\title{
Chlor in dem mittelst Chlorbaryum niedergeschlagenen Baryumsulfat.
}

\author{
Von \\ G. A. Horett und I. H. Duschar.
}

Mit 4 Figuren im Text.

Bei der Herstellung von reinem $\mathrm{BaSO}_{4}$, um den Einflufs der Korngrölse auf die Löslichkeit der $\mathrm{BaSO}_{4}$ klar zu legen, ${ }^{1}$ sind wir der sogenannten ,Okklusion" des Chlorbaryums vomniedergeschlagenen schwefelsauren Baryum entgegengetreten.

Obwohl dieses Problem bei vielen wissenschaftlichen Arbeiten schon behandelt worden ist, liegen doch nur wenige exakte Messungen darüber vor. Die Arbeit von TH. W. Richards und H. G. Parker ${ }^{2}$ ist fast die einzige quantitative, die wir gefunden haben. Die Ursache der kleinen Zahl von quantitativen Ergebnissen über Okklusionsmessungen ist wahrscheinlich in dem Umstand $\mathrm{zu}$ suchen, dals man auf grofse Schwierigkeiten stölst, wenn man die winzigen Mengen des Chlors, (von Spuren bis auf $1 \%$ ), im niedergeschlagenen Baryumsulfat genau bestimmen will.

Natürlich kann man den Niederschlag mit kohlensaurem Natrium aufschliefsen, und das Chlor nachher als Chlorsilber gravimetrisch bestimmen. Dazu braucht man aber sehr reine Reagentien, und die Analyse nimmt sehr viel Zeit in Anspruch. Bei der folgenden einfachen und exakten Methode, das Chlor im schwefelsauren Baryum zu bestimmen, ist es uns gelungen, diese Schwierigkeiten zu umgehen.

1 Zeitschr. phys. Chem. 37 (1901).
2 Amer. Acad. Sci., 31 (1896), 67. 
Schwefelsaures Baryum ist in konzentrierter Schwefelsäure leicht löslich. ${ }^{1}$ Aus der schwefelsauren Lösung wird das Chlor leicht und vollständig als Salzsäure mittelst eines indifferenten Gases ausgetrieben. Die Salzsäure kann man dann in einem Überschufs verdünnter $\mathbf{n} / 100 \mathrm{~g}$ äq. Silbernitratlösung aufnehmen, die in bekannter Menge in zwei Waschflaschen eingefüllt wird. Die Chlorbestimmung geschieht, indem man das überschülsige Silbernitrat zurücktitriert.

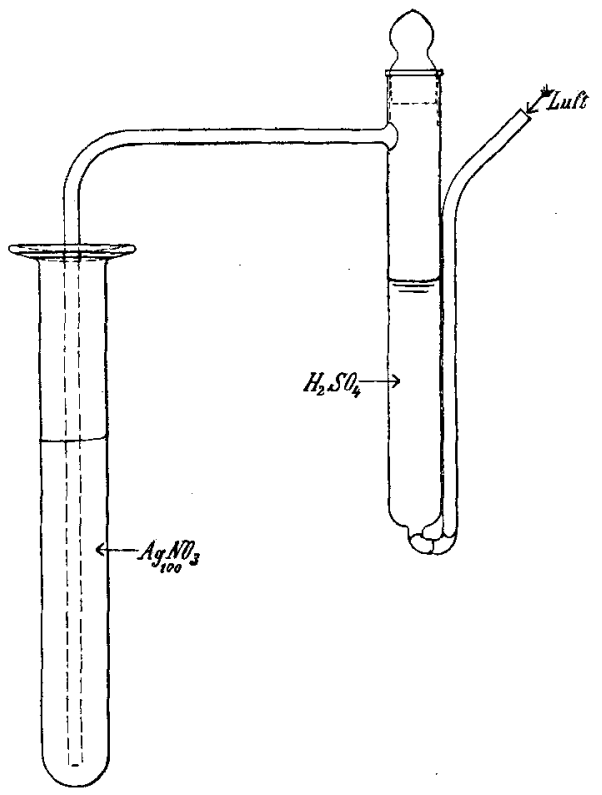

Fig. 1.

Wir finden, dafs ein Strom gereinigter Luft die ganze Menge Salzsäure aus der schwefelsauren Lösung innerhalb zwei Stunden hinausjagt, nachdem der Niederschlag einmal gelöst war. Niemals haben wir Salzsäure in der zweiten Waschflasche konstatieren können. Das Chlor lälst sich auf diese Weise bis auf einige hundertstel Milligramme bestimmen. Die Methode liefert also sehr vertrauenswerte Resultate, wie aus den folgenden Versuchen hervorgeht. Die wirkliche Dauer der Analyse ist übrigens kurz.

Um das Verfahren zu prüfen, wurde in ein kleines Rohr (siehe Fig. 1) eine gewogene Menge gereinigtes umkristallisiertes $\mathrm{BaCl}_{2} \cdot 2 \mathrm{H}_{2} \mathrm{O}$ eingesetzt und hierauf konzentrierte chlorwasserstofffreie Schwefel-

1 Schultz, Pogg. Ann. 133 (1868), 146. 
säure eingegossen. Die zweite Röhre enthielt verdünnte Silbernitratlösung. Das untere Ende des mit Schwefelsäure versetzten Rohres wird mit Wasser erwärmt und ein Strom gereinigter Luft durch das Röhrensystem geführt. Innerhalb zwei Stunden, nachdem das Salz aufgelöst ist, verschwindet die Salzsäure vollständig aus der schwefelsauren Lösung.

Bei den folgenden Analysen hat abwechselnd der Eine von uns das $\mathrm{BaCl}_{2} \cdot 2 \mathrm{H}_{2} \mathrm{O}$ ausgewogen und der Andere die Chlorbestimmung ausgeführt. Das Chlorsilber war in den letzten zwei Versuchen gravimetrisch in einem Goochschen Tiegel bestimmt.

$\begin{array}{cc}\text { Ausgewogenes } & \text { Gefunden, und als } \\ \mathrm{BaCl}_{2} \cdot 2 \mathrm{H}_{2} \mathrm{O}: & \mathrm{BaCl}_{2} \cdot 2 \mathrm{H}_{2} \mathrm{O} \text { berechnet: } \\ 14.65 \mathrm{mg} & 14.64 \mathrm{mg} \\ 14.02 " & 14.09 " \\ 12.66 " & 12.59 " \\ 7.23, & 7.28 " \\ 14.30 " & 14.30 " \\ 22.40 " & 22.39 "\end{array}$

Es sind dieses die ersten auf obige Weise ausgeführten Analysen. Nach den vielen Erfahrungen, die wir in der Zwischenzeit gemacht haben, ergibt die Methode Resultate bis auf $0.01 \mathrm{mg}$. genau.

Benutzt man Chlorbaryum, um schwefelsaures Baryum aus wässerigen Lösungen niederzuschlagen, so enthält der Niederschlag immer mehr oder weniger Chlor. Wir haben sogar bis auf $1 \%$ Chlor im $\mathrm{BaSO}_{4}$-Niedersehlag gefunden. Die Bestimmung von , $\mathrm{SO}_{4}$ " mittelst Chlorbaryums ergibt also Resultate, die immer zu hoch liegen. Wenn aber das Chlor im geglühten Niederschlag bestimmt und als Chlorbaryum berechnet wird, so stimmt das Gewicht des Niederschlages mit der zuerst genommenen Menge „, $\mathrm{SO}_{4}$ “ überein, wie RichaRds gezeigt hat. ${ }^{1}$

Man kann dieses sogenannte „okkludierte“ Chlorbaryum nicht aus dem Niederschlag auswaschen. Auch mittelst Glühen, Auslaugen mit Säuren, und darauf folgendes Auswaschen lälst es sich nicht vollständig entfernen. ${ }^{2}$

1 Proc. Amer. Acad. Sci. 26 (1891), 258.

2 Sregre, Journ. prakt. Chem. 69 (1856), 142. - Rose, Pogg. Ann 113 (1861), 127. - Fresenids, Zeitsehr. analyt. Chem.9 (1870), 52. - Marr, Amer. Joiim. Sei. 41 (1891), 294. 
In der vorliegenden Arbeit wollten wir nun die Menge sowie die Natur dieses so hartnäckig auf ganz besondere Weise zurückgehaltenen Chlorbaryums zu bestimmen versuchen, und zwar in den Fällen, wo schwefelsaures Baryum aus Lösungen bekannter Konzentrationen niedergeschlagen wird.

Eine der ersten Fragen, die uns entgegengetreten, betrifft das Auswaschen des Niederschlages. Alle anhaftende Lösung sowie adsorbierte Salze ${ }^{1}$ müssen aus der Oberfläche des Niederschlages entfernt werden. Das Auswaschen darf aber nicht so weit getrieben werden, dal's etwas rom okkludierten Chlorbaryum aus den Oberflächenschichten entfernt wird. Wegen der grolsen Oberfläche des Niederschlages, die auf dessen feiner Verteilung beruht, ${ }^{2}$ muls man eben diesen Punkt sorgfältig berücksichtigen. Übertriebenes Auswaschen könnte etwas von dem okkludierten Chlorbaryum aus der Oberfläche entfernen. Um diesen Punkt zu prüfen, sind folgende Versuche angestellt worden.

Zwei Millimole (=0.467 g) schwefelsaures Baryum wurden jedesmal niedergeschlagen und der Niederschlag wurde auf ein Asbestfilter (siehe Fig. 2) gebracht. Dieser besteht aus einer durchlöcherten Porzellanscheibe, die in einem $2^{1 / 2} \mathrm{~cm}$ breiten Glasrohr eingebracht wird,

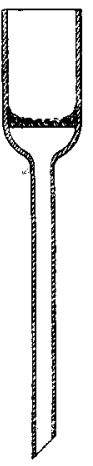

Fig. 2. welches unter der Scheibe ausgezogen ist. Den schmäleren Teil des Rohres führt man durch den Pfropfen einer BUNsenschen FiltrierHasche.

Die bekannte Menge des Niederschlages wird auf diesem Filter aufgesammelt, die Wasserstrahlpumpe in Tätigkeit gesetzt und der Niederschlag wird gut ausgesaugt. Man saugt jedesmal $3 \mathrm{ccm}$ des Waschwassers wieder durch das Filter, damit der Niederschlag immer ganz gleiche Behandlung bekommt. Nun bringt man den Niederschlag nebst Asbest ${ }^{3}$ in den Chlorbestimmungsapparat hinein (siehe Fig. 1) und führt alsbald die Chlorbestimmung aus.

I Ostwald, Anal. Chem. S. 19. - Zawidskr, Zeitschr. phys. Chem. 35 $1900), 77$.

${ }_{2}^{2}$ Niedergeschlagenes schwefelsaures Baryum, welches vom Filter gut zurückgehalten wird, besteht aus Partikelchen vom Durchmesser 1-2 $\mu$. Die Oberfläche von $1 \mathrm{~g} \mathrm{BaSO}$, beträgt also etwa $1.3 \mathrm{~m}^{2}$ resp. $0.80 \mathrm{~m}^{2}$.

${ }^{3}$ Kontrollversuche zeigten dafs der Asbest keinen Fehler bei der Chlorbestimmung verursacht. 
Je nach der Korngrölse der Partikelchen braucht man nur das Auswaschen des Niederschlages verschiedene Male zu wiederholen, um keine Spur von Chlor im Waschwasser konstatieren zu können. Falls der Niederschlag von vornherein grobkörnig war, gentlgen fünf bis sechs Male; bei feinkörnigen Niederschlägen aber muls main mehr als sechs Mal auswaschen. Folgendes Beispiel erläutert diesen Punkt.

Wir setzten zwei Millimole Schwefelsäure zur ChlorbaryumLösung $(v=2 \mathrm{~L}$.) und beim fünften Auswaschen bekamen wir immer noch eine Spur Chlor im Waschwasser, beim sechsten aber gar keine mehr, obwohl der Niederschlag Chlor äquivalent $1.3 \%$ Chlorbaryum enthielt.

Ein anderer Versuch bestand darin, dafs chlorfreies schwefelsaures Baryum aus Barytlösung mit Schwefelsäure niedergeschlagen und durch geeignete Behandlung im grobkörnigen Zustande erhalten wurde. Dann wurde der Niederschlag während fünf Minuten mit Chlorbaryum-Lösung $(v=1 \mathrm{~L})$ geschüttelt. Erst beim siebenten Auswaschen war das Waschwasser chlorfrei und der Niederschlag enthielt auch keine Spur Chlor mehr. Das Auswaschen des Niederschlages, der okkludiertes Chlorbaryum enthält, unterscheidet sich also nicht merklich vom Auswaschen des chlorfreien. Deswegen schliefsen wir, dals kein merklicher Verlust an Chlor stattfindet bei unserem Verfahren, den Niederschlag auszuwaschen. Die Mutterlauge sowie alles adsorbiertes Chlorbarium wird aber vollständig aus dem Niederschlag entfernt.

Eine Schwefelsäurelösung $(v=10 \mathrm{~L}$.) wurde aufs sorgfältigste hergestellt und genau $20 \mathrm{ccm}$ dieser Lösung wurden bei jedem Versuch angewendet. Man setzt diese Schwefelsäure-Lösung sehr langsam der Chlorbaryum-Lösung hinzu und gleichzeitig eine andere ,korrigierende,, Lösung, um die Konzentration des Chlorbaryums konstant zu erhalten.

Der Versuch wird in einer breithalsigen Jenaerflasche ausgeführt, welche auf einem Wasserbad bei $100^{\circ} \mathrm{C}$ erwärmt ist. Der Inhalt dieser Flasche wird mit einem mittelst Motors getriebenen Rührwerk umgerührt.

Man setzt die $\mathrm{H}_{2} \mathrm{SO}_{4}$-Lösungen ( $v=10 \mathrm{~L}$. bei allen Versuchen) langsam den $\mathrm{BaCl}_{2}$-Lösungen hinzu, damit das schwefelsaure Baryum in Gegenwart einer bestimmten konstant erhaltenen Konzentration von Chlorbaryum entsteht, während die $\mathrm{H}_{2} \mathrm{SO}_{4}$-Konzentration sehr klein bleibt, gerade in dem Punkte, wo der Niederschlag sich 
bildet. Um die Konzentration des Chlorbaryums immer konstant zu erhalten, fügt man gleichzeitig mit der Schwefelsäure, aber auf der entgegengesetzten Seite der Jenaerflasche die korrigierende Lösung hinzu, die Chlorbaryum nebst Baryt enthält, falls man $\mathrm{HCl}$-Konzentration $=0$, oder Chlorbaryum nebst Salzsäure, wenn man eine konstante Konzentration von Chlorbaryum und Salzsäure wünscht. Zur Zeit des Entstehens des Niederschlages, sowie nachher, wird das System kräftig umgerührt.

Nun wird der Niederschlag auf dem Filter gesammelt, ausgewaschen, wie oben angegeben, und, ohne zu trocknen, in den Chlorbestimmungsapparat eingebracht.

Wir fanden alsbald, dafs der Chlorgehalt des Niederschlages von der Dauer des Umrührens, nachdem der Niederschlag schon gebildet wurde, abhängt.

Bei den folgenden Versuchen wurden jedesmal $20 \mathrm{ccm} \mathrm{H}_{2} \mathrm{SO}_{4^{-}}$ Lösung zu $\mathrm{BaCl}_{2}$-Lösung $(v=4 \mathrm{~L}$.) hinzugesetzt und während verschiedener Zeiten war das ganze System umgerührt. ${ }^{1}$ Die nachstehende Tabelle erläutert die Versuchsergebnisse.

Dauer des

Umrührens :

11 Stunden

$52 \quad$,

$160 \quad$,
Mole Chlor $\left(\mathrm{Cl}_{2}\right)$ in 100 Mole

Schwefelsäure:

(a) 1.38

(b) 1.32

(a) 1.14

(b) 1.17

(a) 1.06

(b) 1.07

Diese Resultate geben ein gewisses Zeitelement zu erkennen, indem der Chlorgehalt des Niederschlages sich mit der Dauer des Umrührens vermindert. Nun ist der $\mathrm{BaSO}_{4}$ Niederschlag im Moment des Entstehens sehr feinkörnig, wird aber in Berübrung mit der Lösung grobkörnig. ${ }^{2}$ Die Änderung im Chlorgehalt des Niederschlages verlänft dieser Änderung in der Grölse der Partikelchen

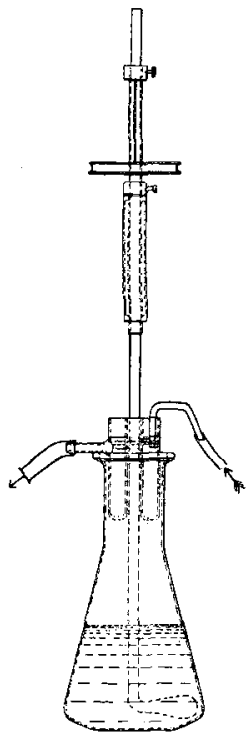

Fig. 3.

1 Wir haben kleine Wasserkühler (siehe Fig. 3) im Halse der Jenaerflasche angebracht, um eine Verminderung der Konzentration durch Verdunstung während des Umrührens zu vermeiden. Der Rührer geht durch die Mitte des Wasserkühlers.

${ }^{2}$ Holett, Zeitschr. phys. Chem. 37 (1901), 385.

Z. anorg. Chem. Bu. 40. 
parallel. Je konzentrierter die Chlorbaryumlösung, um so langsamer erreichen die Partikelchen ihre maximale Grölse während des Umrührens. Bei diesen Lösungen hat es länger gedauert, bis der minimale Chlorgehalt erreicht ist. In Gegenwart von Salzsäure wachsen sie aber schneller bis zur maximalen Grölse, ${ }^{1}$ und der minimale Chlorgehalt wird in diesem Fall eher erreicht als im vorigen Fall.

Die grobkörnigen niedergeschlagenen Partikelchen haben eine viel kleinere Gesamtoberfläche als die feinkörnigen. Nun merkt man, dafs die Verminderung des Chlorgehaltes auch der Verminderung der Gesamtoberfläche parallel geht. Aber unsere Versuche in bezug auf das Auswaschen des Niederschlages führen zu der Meinung, dafs der Prozent Chlorgehalt nicht eine Funktion der Oberfläche des Niederschlages ist. Wir werden später auf diesen Punkt zurückkommen.

Wir haben die Lösungen immer so lange umgerührt, bis der Niederschlag grobkörnig geworden war, was von der Dauer des Absetzens leicht zu ersehen ist, wenn der Motor einmal abgestellt wird. Wir nehmen nun an, dafs dann der Niederschlag fast im Gleichgewicht mit der Lösung steht.

Zunächst wurde eine Reihe von Versuchen angestellt, um den Einflufs von verschiedenen Konzentrationen des Chlorbaryums ins klare zu bringen. Bei dieser Versuchsreihe enthält die korrigierende Lösung immer Baryt nebst Chlorbaryum, damit die $\mathrm{BaCl}_{2}$-Konzentration konstant, und die HCl-Konzentration gleich Null wird. Jedesmal wurden $20 \mathrm{ccm}$ Schwefelsäurelösung $(v=10 \mathrm{~L}$.), den verschiedenen Chlorbaryumlösungen hinzugesetzt und das Ganze wurde während verschiedenen Zeiten umgerührt. Es ergibt sich folgendes:

\begin{tabular}{|c|c|c|c|}
\hline Konzentration & $\begin{array}{l}\text { Dauer des } \\
\text { Umrührens }\end{array}$ & $\begin{array}{c}\text { Mole } \mathrm{Cl}_{2} \text { in } \\
100 \text { Mole } \mathrm{BaSO}_{4}\end{array}$ & $\begin{array}{l}\text { Prozent Chlor } \\
\text { im Niederschl. }\end{array}$ \\
\hline $\mathrm{BaCl}_{2}(v=1 \mathrm{~L})$. & 240 Stunden & (a) 2.10 & 0.64 \\
\hline $\mathrm{BaCl}_{2}(v=1 \mathrm{~L})$. & 240 & (b) 2.21 & 0.67 \\
\hline $\mathrm{BaCl}_{2}(v=2$ L. $)$ & 140 & (a) 1.47 & 0.46 \\
\hline $\mathrm{BaCl}_{2}(v=2 \mathrm{~L})$. & 140 & (b) 1.54 & 0.48 \\
\hline $\mathrm{BaCl}_{2}(v=4$ L. $)$ & 160 & (a) 1.06 & 0.32 \\
\hline $\mathrm{BaCl}_{2}(v=4 \mathrm{~L})$ & 160 & (b) 1.07 & 0.33 \\
\hline $\mathrm{BaCl}_{2}(v=8 \mathrm{~L})$. & 120 & (a) 0.853 & 0.25 \\
\hline $\mathrm{BaCl}_{2}(v=8$ I. $)$ & 120 & (b) 0.870 & 0.265 \\
\hline
\end{tabular}

1 Vergl. MARR, Amer. Journ. Sei. 41 (1891), 288. 
Bei vielen anderen Versuchen wurden genau die obigen Konzentrationen angewendet; nur war die Dauer des Umrührens kürzer, aber alle diese zeigen auch das "Zeitelement".

Aus der angegebenen Versuchsreihe geht nun klar hervor, dals es keine direkte Proportionalität zwischen $\mathrm{BaCl}_{2}$-Konzentration und der Menge des im Niederschlag enthaltenen Chlors gibt.

Es war immer schwierig, bei verdünnten Lösungen irgend ein Resultat zu wiederholen. Die Versuche mit Chlorbayrum

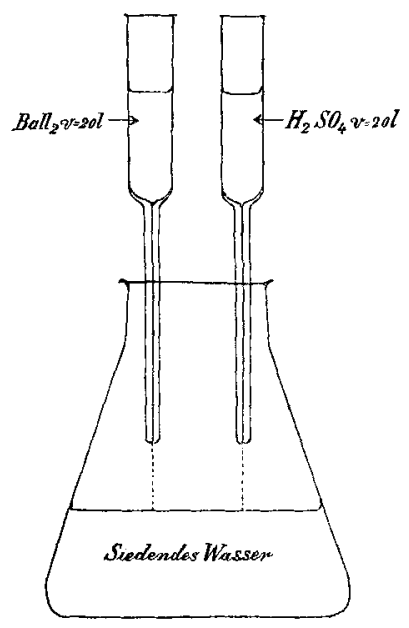

Fig. 4.

$(v=16 \mathrm{~L}$.) ergeben z. B. eine Schwankung von 0.16 bis $0.35 \%$ Chlor im Niederschlag. Bei diesen und auch bei den verdünnteren Lösungen vermindert sich der Prozent-Chlorgehalt gar nicht der Verdünnung proportional. Folgender Versuch wurde auch ausgeführt. Auf der einen Seite einer $250 \mathrm{ccm}$ kochendes Wasser enthaltenden Flasche wurden $20 \mathrm{ccm} \mathrm{H}_{2} \mathrm{SO}_{4}(v=10 \mathrm{~L}$.), auf der entgegengesetzten Seite $20 \mathrm{ccm} \mathrm{BaCl}{ }_{2}(v=10$ L.) langsam hineingelassen. Dazu benutzten wir Tropfröhre ${ }^{1}$ (siehe Figur 4), welche 45 Minuten brauchten, um $20 \mathrm{ccm}$ abzuliefern. Die Konzentrationen des eingebrachten $\mathrm{BaCl}_{2}$ und $\mathrm{H}_{2} \mathrm{SO}_{4}$ werden unter diesen Umständen niemals das Löslichkeitsprodukt von $\mathrm{BaSO}_{4}$ sehr übersteigen, etwa

1 Solche Tropfröhren, welche zu gleicher Zeit dieselbe Menge von wässerigen Lösungen abliefern, sind leicht zu konstruieren aus gewöhnlichen Probierröhren mit kapillaren Ansätzen. Die Lösungen Alelsen sehr gleichmäfsig von den Röhren ab und beliebig langsạm durch geeignete Wahl der Kapillare. 
$v=10.000 \mathrm{~L}$. Der $\mathrm{BaSO}_{4}$-Niederschlag war nun schön kristallisiert, sah wie Sand aus, enthielt aber $0.03 \%$ Chlor.

Jetzt wollten wir den Einfluls von Salzsäure auf den Chlorgehalt des $\mathrm{BaSO}_{4}$-Niederschlages studieren. Bei diesen Versuchen wurden $20 \mathrm{ccm}$ Schwefelsäure zu einer Lösung hinzugesetzt, die Chlorbaryum nebst Salzsäure enthält, um die Konzentration von Chlorbaryum und von Salzsäure konstant zu erhalten.

$\begin{array}{llcc}\text { Konzentration } & \begin{array}{c}\text { Dauer des } \\ \text { Unrührens }\end{array} & \begin{array}{c}\mathrm{Mole}_{2} \text { in } \\ 100 \text { Mole BaSO }\end{array} & \begin{array}{c}\text { Prozent } \mathrm{Cl}_{2} \text { im } \\ \text { Niederschlag }\end{array} \\ \mathrm{BaCl}_{2}(v=4 \mathrm{~L} .) & 50 \text { Stunden } & \text { (a) } 0.487 & 0.15 \\ \mathrm{HCl}(v=2 \mathrm{~L} .) & 50 \quad, & \text { (b) } 0.514 & 0.16 \\ \mathrm{BaCl}_{2}(v=4 \mathrm{~L} .) & 112 \quad, & \text { (a) } 0.63 & 0.19 \\ \mathrm{HCl}(v=4 \mathrm{~L} .) & 112 \quad, & \text { (b) } 0.72 & 0.22\end{array}$

Man merkt, dafs der Prozent-Chlorgehalt des Niederschlages sich mit der $\mathrm{HCl}$-Konzentration, bei konstanter $\mathrm{BaCl}_{2}$-Konzentration, vermehrt, und zwar nicht im Verhältnis der $\mathrm{HCl}$-Konzentrationen. Weiter merkt man, dafs der Prozentgehalt an Chlor bei Gegenwart von Salzsäure bedeutend kleiner ist, als in den Fällen, wo der Niederschlag aus neutraler $\mathrm{BaCl}_{2}$ - Lösung, $(v=4$ L.) vergl. S. 202, gebildet worden ist.

Richards und Parken ' haben das umgekehrte gefunden, nämlich, dafs Salzsäure den Chlorgehalt des Niederschlages vermindert. Sie setzten aber Salzsäure der Schwefelsäure hinzu und bestimmten das Chlor im geglühten Niederschlag. Es ist also nicht nötig, anzunehmen, dafs unsere Resultate gegen jene von Rrchakds und Parker deuten. Sie zeigen wohl, dafs der Einflufs von Salzsäure sehr kompliziert ist. Wir beabsichtigen den Einflufs von Salzsäure auf das im niedergeschlagenen schwefelsauren Baryum vorhandene Chlor später zu studieren.

Erst jetzt haben wir eine wichtige Tatsache entdeckt, welche, soweit wir wissen, bisher nicht beobachtet worden ist. Beim Trocknen bleibt das Gewicht des $\mathrm{BaSO}_{4}$-Niederschlages unterhalb $300^{\circ} \mathrm{C}$ ganz unverändert. Sobald aber die Temperatur etwa $325^{\circ} \mathrm{C}$. übersteigt, findet eine merkliche Abnahme im Gewicht statt, welche dauert, bis etwa $600^{\circ} \mathrm{C}$. erreicht sind. Von dieser letzten Temperatur an bleibt dann das Gewicht ganz konstant.

\footnotetext{
1 Proc. Amer. Acad. Sei. 31 (1896), 74.
} 
Erhitzt man den $\mathrm{BaSO}_{4}$-Niederschlag in einem engen Röhrchen, so gibt er eine farblose Flüssigkeit ab, welche sauer reagiert und mit salpetersaurem Silber einen weifsen Niederschlag bildet, aber keinen mit Chlorbaryum. Der $\mathrm{BaSO}_{4}$-Niederschlag verliert also Salzsäure und Wasser zwischen etwa $300^{\circ} \mathrm{C}$. und Rotglühhitze. In dem geglühten $\mathrm{BaSO}_{4}$-Niederschlag bleibt aber immer noch Chlor bestehen. Der Verlust an Chlor, welches als Salzsäure entwichen ist, stellt sich immer viel grölser dar, als die Menge des zurückgehaltenen Chlors. Wir haben bis jetzt keine stöchiometrische Beziehung zwischen diesem ,fixen" und dem ",lüchtigen" Chlor finden können.

Folgende Versuche wurden zunächst angestellt, um diese eben besprochene Tatsache noch weiter zu erläutern. $100 \mathrm{ccm}$ Schwefelsäure $(v=10$ L.) wurden sehr langsam einer Chlorbaryumlösung $(v=4 \mathrm{~L}$.) hinzugesetzt und das ganze wurde 108 Stunden umgerührt. Eine korrigierende Lösung wurde nicht hinzugetan, der $\mathrm{BaSO}_{4}$-Niederschlag bildete sich in Gegenwart von Chlorbaryum und einer sich immer vermehrenden Menge Salzsäure. Dann wurde der Niederschlag gründlich ausgewaschen und bei $140^{\circ} \mathrm{C}$. getrocknet. In 100 Molen des $\mathrm{BaSO}_{4}$-Niederschlages haben wir $0.81 \mathrm{Mole}^{\mathrm{Cl}_{2}}$, also $0.25 \%$ Chlor gefunden. Beim Glühen verlor der Niederschlag noch $1.36 \%$ Wasser, sowie $0.20 \%$ Chlor, da der geglühte Niederschlag nur $0.05 \%$ Chlor enthielt.

Jetzt haben wir Schwefelsäure einer kochenden Chlorbaryumlösung ( $v=4$ L.) sehr langsam hinzugesetzt und gleichzeitig eine korrigierende Lösung, die Chlorbaryum nebst Baryt enthielt, zugetan, um die Iösung, worin der Niederschlag sich bildete, immer ein bischen alkalisch zu halten. In diesem Falle enthielten 100 Mole des $\mathrm{BaSO}_{4}$-Niederschlages 0.63 Mole Salzsäure, 7.8 Mole Wasser, 0.21 Mole vom fixen Chlor; d. h. $0.096 \%$ fü̈chtiges Chlor, $0.062 \%$ fixes Chlor und $0.60 \%$ Wasser. Der $\mathrm{BaSO}_{4}$-Niederschlag aus neutraler oder sogar alkalischer Lösung gibt also Salzsäure und Wasser beim Glühen ab.

Bei noch einem weiteren Versuch wurde Chlorbaryum der schwefelsauren Lösung bei $25^{\circ} \mathrm{C}$. zugesetzt und 21 Tage umgerührt, also das umgekehrte von allen vorigen Versuchen. Jetzt wurden in $100 \mathrm{Molen} \mathrm{BaSO}_{4}$-Niederschlag 1.67 Mole Salzsäure, 3.6 Mole Wasser, 0.18 Mole Chlor gefunden; also $0.25 \%$ Chlor als Salzsäure, $0.28 \%$ Wasser und $0.054 \%$ rom fixen Chlor.

Wie aus den verschiedenen Versuchsbedingungen zu erwarten ist, stimmen die Mengen rom fixen und flüchtigen Chlor unterein- 
ander bei den drei Versuchen nicht überein. Sie zeigen aber ganz klar, dafs der $\mathrm{BaSO}_{4}$-Niederschlag immer flüchtiges sowie fixes Chlor enthält, wie auch die Schwefelsäure- und Chlorbaryumlösungen zusammengebracht seien. Der gut ausgewaschene Niederschlag gibt dementsprechend Salzsäure beim Glühen ab, obwohl er aus alkalischer Lösung gebildet wird, worin $\mathrm{HCl}-$ Konzentration $=\mathrm{O}$ ist.

Es entsteht nun die Frage: Ist dieser Niederschlag, welcher Chlor als Salzsäure beim Glühen abgegeben hat, neutral?

Wenn eine bekannte Menge $\mathrm{SO}_{4}$ " mit Chlorbaryum bestimmt wird, so wiegt, wie jedermann weifs, das geglühte $\mathrm{BaSO}_{4}$ mehr als zu erwarten ist. Bei seiner Untersuchung des Atomgewichtes von Kupfer hat Richards ${ }^{1}$ das $\mathrm{SO}_{4}{ }^{\prime \prime}$ in $\mathrm{CuSO}_{4} .5 \mathrm{H}_{2} \mathrm{O}$ zu messen gesucht, indem er mit Chlorbaryum das $\mathrm{SO}_{4}^{\prime \prime}$ als schwefelsaures Baryum niedergeschlagen hat und das "okkludierte" Chlorbaryum in Rechnung gebracht auf die Weise, dafs er das Chlor im geglühten Niederschlag bestimmte. Der aus $3.1902 \mathrm{~g} \mathrm{CuSO}_{4} .5 \mathrm{H}_{2} \mathrm{O}$ erhaltene $\mathrm{BaSO}_{4}$ Niederschlag hat nach dem Glühen $2.9967 \mathrm{~g}$ gewogen, in welchem $0.23 \%$ Chlor, d. h. 0.0206 g Chlorbaryum gefunden wurde. Es blieb also nur $2.9761 \mathrm{~g}$ schwefelsaures Baryum, während die berechnete Menge $2.9828 \mathrm{~g}$ beträgt. Da das schwefelsaure Baryum sowie das Chlorsilber im Waschwasser nicht bestimmt wurde, so ist die Übereinstimmung genügend. Eine spätere Untersuchung von RicharDs und PARKER über die Okklusion des Chlorbaryums vom schwefelsauren Baryum ${ }^{2}$ führt zu dem Schlufs, "that this error due to occlusion may be corrected with great exactness by determining the amount of chlorine held by the precipitate and subtracting the corresponding amount of baryum chloride from the total weight of the precipitate".

Obwohl dieser von Richards und Panker aus seinen Versuchsergebnissen gezogene Schlufs gerechtfertigt ist, schien es uns doch ratsam, noch weitere Beweise aufzusuchen. Zunächst haben wir schwefelsaures Baryum hergestellt, wie RICHARDs angibt: wir setzten also Chlorbaryum einer kochenden, mit Salzsäure stark angesäuerten Kupfersulfatlösung hinzu. Der sorgfältig ausgewaschene und bei $140^{\circ} \mathrm{C}$. getrocknete Niederschlag enthielt $0.51 \%$ Chlor. Beim Erhitzen bis etwa $650^{\circ} \mathrm{C}$. nahm das Gewicht um $1.15 \%$ ab. Es blieb nur $0.26 \%$ Chlor zugegen, und beim Glühen in einem

1 Proe. Amer. Acad. Sci. 26 (1891), 258. - Z. anorg. Chem. 1, 150. $18 \overline{.}$.

2 Proc. Amer. Acad. Sei. 31 (1896), 77. 
Strom reiner Luft fand noch eine weitere Abnahme des Chlors statt. Der Rückstand zeigte sich nun gegen Phenolphtaleinlösung etwas alkalisch. Es schien als ob der Niederschlag beim starken Glühen in einem Luftstrom etwas fixes Chlor verloren hätte.

Nun haben wir Versuche ausführen wollen, um den Gewichtsverlust bei verschiedenen Temperaturen kennen zu lernen. Zum Kontrollversuch stellten wir chlorfreies schwefelsaures Baryum dar auf die Weise, dals gleichzeitig Schwefelsäure- $(v=10 \mathrm{~L}$.) und Barytlösung ( $v=10 \mathrm{~L}$.), sehr langsam in kochendes Wasser eingelassen wurden. Dabei war immer Schwefelsäure im kleinen Überschufs zugegen und Kohlensäure wurde fern gehalten.

Aufserdem haben wir kristallinisches ${ }^{1}$ schwefelsaures Baryum präpariert, welches ausgewaschen und während mehrerer Tage unter Wasser stehen gelassen wurde.

Diese Kriställchen wurden dann bei $150^{\circ} \mathrm{C}$. getrocknet. Sie enthielten $0.22 \%$ Chlor.

Wir breiteten $2.3845 \mathrm{~g}$ der Diffusionskristalle in einer kleinen Platinschale aus und in einer zweiten gleichen Platinschale $1.9396 \mathrm{~g}$ des chlortreien schwefelsauren Baryums. Die zwei Proben wurden nun gleichartig in einem elektrischen Ofen erhitzt, dessen Heizraum $6.5 \mathrm{~cm}$ breit und $30 \mathrm{~cm}$ tief war. Die Schalen werden mit Platindrähten mitten im Heizraum aufgehängt. Um die Temperatur des Heizraums zu messen, benutzten wir ein von der Reichsanstalt geprüftes Thermoelement, dessen E.M.K. durch Kompensation bis auf \pm 0.000005 Volt bestimmt wurde. Mittelst eines grofsen Rbeostaten konnten wir irgend eine Temperatur bis auf $1200^{\circ} \mathrm{C}$. innerhalb einiger Grade beliebig lang einhalten. Während des Erhitzens wird immer ein langsamer Strom $\mathrm{CO}_{2}$-freier Luft durch den Heizraum geführt. Folgende Tabelle gibt diese Versuchsergebnisse an.

1 Dieses Präparat war anf folgende Weise hergestellt. Auf der einen Seite einer sehr grofsen $60 \mathrm{~cm}$ breiten Kristallisierschale wird eine kleine, gesättigte Chlorbaryumlösung enthaltende Schale, auf der anderen eine gleiche, mit konzentrierter Schwefelsäure gefüllte, Schale eingesetzt. Diese zwei kleinen Schalen stehen in zwei grölseren aber niedrigen Schalen. Nun giefst man Wasser sehr langsam in die grofse Schale hinein, bis die kleineren mit Wasser eben aufgedeckt sind. Als Schutz gegen Staub ans der Luft dienen grofse Glasplatten: Bei dem langsamen Diffundieren von den Chlorbaryum- und Schwefelsäurelösungen ineinander, entstehen kleine aber ganz klare federartige Kriställchen von schwefelsaurem Baryum, welche eine bedeutende Menge Chlor enthalten. 


\begin{tabular}{|c|c|c|c|c|c|}
\hline \multicolumn{2}{|c|}{ Reines $\mathrm{BaSO}_{4}$} & \multirow{2}{*}{$\begin{array}{l}\text { Tempe- } \\
\text { ratur }\end{array}$} & \multirow{2}{*}{$\begin{array}{c}\text { Dauer des } \\
\text { Erhitzens } \\
\text { in Stunden }\end{array}$} & \multicolumn{2}{|c|}{ Diffusionskristalle } \\
\hline Gewicht in $g$ & Verlust v. $1 \mathrm{~g}$ & & & Gervicht in $\mathrm{g}$ & Verlust v. $1 \mathrm{~g}$ \\
\hline 1.9396 & & 150 & 12 & 2.3845 & \\
\hline 1.9372 & 0.00124 & 310 & $2^{1 / 2}$ & 2.3679 & 0.00700 \\
\hline \multirow[t]{2}{*}{1.9265} & \multirow[t]{2}{*}{0.00036} & \multirow[t]{2}{*}{370} & \multirow[t]{2}{*}{13} & 2.3529 & 0.00640 \\
\hline & & & & \multicolumn{2}{|c|}{ Probe ausgenommen } \\
\hline 1.9366 & - & 430 & $4^{1 / 2}$ & 1.8942 & 000080 \\
\hline \multirow[t]{2}{*}{1.9359} & \multirow[t]{2}{*}{0.00036} & \multirow[t]{2}{*}{510} & \multirow[t]{2}{*}{12} & 1.8895 & 0.00248 \\
\hline & & & & \multicolumn{2}{|c|}{$\begin{array}{l}\text { Probe ausgenommen } \\
1.4487\end{array}$} \\
\hline $1.933 \check{0}$ & - & 565 & $2^{1 / 2}$ & 1.4473 & 0.00055 \\
\hline 1.9353 & - & 650 & $2^{1 / 2}$ & 1.4463 & 0.00069 \\
\hline 1.9356 & - & \multirow[t]{2}{*}{650} & \multirow[t]{2}{*}{12} & $1.44 n 1$ & - \\
\hline \multicolumn{2}{|c|}{ Probe alusgenommen } & & & \multicolumn{2}{|c|}{ Probe ausgenommen } \\
\hline 1.8038 & & 785 & 2 & 1.0093 & \\
\hline 1.8039 & - & 800 & 2 & 1.0093 & - \\
\hline 1.8035 & 0.00022 & 944 & 2 & 0.0089 & 0.00040 \\
\hline 1.8033 & 0.00011 & 900 & 3 & 0.0089 & - \\
\hline 1.8032 & 0.00014 & 957 & 3 & 0.0085 & 0.00040 \\
\hline
\end{tabular}

Aus obiger Tabelle ersieht man, dafs reines chlorfreies schwefelsaures Baryum, welches zum konstanten Gewicht bei $140^{\circ} \mathrm{C}$. getrocknet ist, immer noch Wasser enthält, da das Gewicht bis zu $500^{\circ} \mathrm{C}$. immer abnahm. Von $500^{\circ} \mathrm{C}$. an bleibt es dann konstant. Die kleinen Gewichtsänderungen, die man bei $944^{\circ} \mathrm{C}$. bemerkt, hängen nicht von einer Zersetzung des schwefelsauren Baryums ab, wie wir später zeigen werden.

Die Diffussionskristalle zeigen aber ein ganz anderes Verhalten. Ein merklicher Gewichtsverlust lälst sich bis etwa $650^{\circ} \mathrm{C}$. erkennen, welcher sich aus $0.18 \%$ Chlor, das als Salzsäure entwichen ist, und $1.13 \%$ Wasser aus der Differenz zusammensetzt. Das Gewicht bleibt bei $650^{\circ} \mathrm{C}$. ganz konstant, und der Rückstand reagiert gar nicht alkalisch, wenn er angefeuchtet und mit Phenolphtaleinlösung geprüft wird. Er enthält $0.14 \%$ Chlor.

Zwischen 650 und $900^{\circ} \mathrm{C}$. findet kein Gewichtsverlust statt: also der Chlorgehalt bleibt unverändert, aber von $900^{\circ}$ C. an, wenn in $\mathrm{CO}_{2}$-freier Luft erhitzt, nimmt das Gewicht ab. Etwas 
fixes Chlor entweicht, und der Rückstand reagiert dann gegen Phenolphtaleinlösung alkalisch. Nach 5 Stunden bei $950^{\circ} \mathrm{C}$. war nur $0.05 \%$ Chlor im Rückstand geblieben und nach weiteren 3 Stunden bei $1050^{\circ} \mathrm{C}$. und nach weiteren 2 Stunden bei $1100^{\circ} \mathrm{C}$. war nur noch $0.01 \%$ Chlor übrig. Das niedergeschlagene $\mathrm{BaSO}_{4}$ verhält sich beim Erhitzen diesem kristallinischen ganz ähnlich.

Aus diesen Resultaten mit den Diffusionskristallen schlielst man, dals der $\mathrm{BaSO}_{4}$-Niederschlag zwischen $300^{\circ} \mathrm{C}$. und $650^{\circ} \mathrm{C}$. seinen ganzen Gehalt an Wasser und flüchtiges Chlor (Salzsäure) verliert. Von $650^{\circ} \mathrm{C}$. an bis $900^{\circ} \mathrm{C}$. besteht der Rückstand aus schwefelsaurem Baryum und Chlorbaryum, da er immer noch Chlor enthält und auch neutral reagiert. Von $900^{\circ} \mathrm{C}$. an findet eine weitere Abnahme an Chlor statt und der Rückstand reagiert jetzt alkalisch. Wir haben nun beobachtet, dafs reines schwefelsaures Baryum, welches in $\mathrm{CO}_{2}$-freier Luft erhitzt wird, erst bei $1100^{\circ} \mathrm{C}$. eine merkliche Abnahme im Gewicht erfährt. Nach 4 Stunden bei $1125^{\circ} \mathrm{C}$. und weiteren $2^{1} / 2$ Stunden bei $1230^{\circ} \mathrm{C}$. betrug der Verlust $1.3 \mathrm{mg}$ per Gramm und der Rückstand hat dann alkalisch reagiert. Nebenbei finden wir, dals die Platingefälse, die in einem Luftstrom bei $1000^{\circ} \mathrm{C}$. erhitzt werden, einen Gewichtsverlust erleiden. Eine Platinschale, die zuerst $9.637 \mathrm{~g}$ gewogen hat, verlor nach 4 Stunden bei $1125^{\circ} \mathrm{C} .1 .4 \mathrm{mg}$. Wir haben bei den obigen Versuchen über $1100^{\circ} \mathrm{C}$. beobachtet, dals eine metallische Schicht sich auf dem Porzellandeckel des elektrischen Ofens bildete, welche sich als Platin erweist. Wir gedenken später weitere Versuche über diesen Gegenstand anzustellen.

Um noch weitere Beweise der Neutralität des zwischen $650^{\circ} \mathrm{C}$. und $900^{\circ} \mathrm{C}$. geglühten Niederschlages zu bekommen, stellten wir eine Schwefelsäurelösung aus sorgfältig gereinigten Ausgangsstoffen her, welche gegen Bernsteinsäure geprüft wurde, sowie auch gegen kohlensaures Natrium, das aus Natrium in einem silbernen Gefäls präpariert wurde. Bei $23.5^{\circ} \mathrm{C}$. hatte diese Schwefelsäurelösung die Verdünnung $v=9.944 \pm 1 \mathrm{~L}$., welche die Grenzen der Einstellungsgenauigkeit mit den obigen Reagentien darstellt.

Von dieser schwefelsauren Lösung bei $23.5^{\circ} \mathrm{C}$. wurden $50 \mathrm{ccm}$ einem Gemisch von Chlorbaryum ( $v=2 \mathrm{~L}$.) und Salzsäure $(v=0.2 \mathrm{~L}$.) zugesetzt. Der entstandene Niederschlag wurde dann sorgfältig ausgewaschen und das schwefelsaure Baryum aus dem Waschwasser wiedergewonnen.

Der so erhaltene Niederschlag wurde nun bei etwa $700^{\circ} \mathrm{C}$. 
geglüht und hatte dann das Gewicht $1.1884 \mathrm{~g}$. Er enthielt $3.96 \mathrm{mg}$ Chlor, entsprechend $11.63 \mathrm{mg}$ Chlorbaryum. Es ergibt sich also $1.1768 \mathrm{~g}$ schwefelsaures Baryum, während die berechnete Menge $1.1740 \mathrm{~g}$ beträgt.

Wir betrachten die Übereinstimmung zwischen dem gefundenen und berechneten Gewicht als befriedigend, da sich sämtliche Fehler der sehr kleinen Differenz $11.63 \mathrm{mg}$ hinzusetzen. Wir dachten nun, dafs Chlorbaryum sich als sehr geeignet zur Basis für weitere Versuche erweisen würde. Das chemische, reine Chlorbaryum wurde umkristallisiert, ${ }^{1}$ in einem Platingefäls geschmolzen, dann wieder aufgelöst, durch Asbest filtriert, aus dieser Lösung mittels Alkohols ausgeschieden, noch einmal in Leitfähigkeitswasser aufgelöst und wieder aus dieser Lösung umkristallisiert. Dabei wurden immer gut ausgedämpfte Jenaer Flaschen benutzt. Das fein kristallinische Präparat wurde jetzt zu konstantem Gewicht über teilweise entwässertes $\mathrm{BaCl}_{2} \cdot 2 \mathrm{H}_{2} \mathrm{O}$ getrocknet und dann in Glasröhren zwecks Auf bewahrung eingeschmolzen. Aus mehreren gut übereinstimmenden Versuchen, bei welchen das Salz zuletzt in einem mit trockenen Salzsäuredämpfen erfültten Raum erhitzt wurde, ergab sich der Wassergehalt zu $14.780 \%$ (auf dem luftleeren Raum reduziert). $2.5185 \mathrm{~g}$ dieses $14.780 \%$ Wasser enthaltenden Salzes, entsprechend $2.4054 \mathrm{~g} \mathrm{BaSO}_{4}$, wurden mit überschüssiger Schwefelsäure niedergeschlagen und der Niederschlag wurde bei $700^{\circ} \mathrm{C}$. geglüht bis zum konstanten Gewicht $2.3984 \mathrm{~g}$. Wir haben also ein um $7 \mathrm{mg}$ zu kleines Gewicht bekommen, welches $57.9 \mathrm{mg}$ Chlorbaryum im Niederschlag entspricht. Aus der Analyse finden wir nun $19.1 \mathrm{mg}$ Chlor, entsprechend $56.3 \mathrm{mg}$ Chlorbaryum.

Bei noch einem zweiten Versuch haben wir $2.1568 \mathrm{~g}$ Chlorbaryum, entsprechend $2.4039 \mathrm{~g} \mathrm{BaSO}_{4}$ auf gleiche Weise niedergeschlagen. Der bei $700^{\circ} \mathrm{C}$. geglühte Niederschlag hat $2.3982 \mathrm{~g}$ gewogen, also $5.7 \mathrm{~g} \mathrm{zu}$ wenig. Dieser Unterschied entspricht $47.2 \mathrm{mg}$ Chlorbaryum. Der Chlorgehalt des Niederschlages beträgt $19.3 \mathrm{mg}$ Chlor, $57.2 \mathrm{mg} \mathrm{BaCl}$.

Wir haben bei diesen Versuchen die grölste Sorgfalt angewendet, - so z. B. bei allen Operationen entweder Platingefäfse oder Jenaer Glasgefäfse benutzt, immer das schwefelsaure Barium im Waschwasser wieder gewonnen, die Gewichte auf dem luftleeren Raum reduziert, - da irgend ein Fehler bei einer Operation im

1 Vergl. RichaRDs, Z. anorg. Chem. 6 (1894), 95. 
Endresultat viel vergröfsert zum Vorschein kommt. Daher betrachten wir die Resultate als befriedigend. Aus den Resultaten glauben wir gezeigt ' $\mathrm{zu}$ haben, dafs der bei $700^{\circ} \mathrm{C}$. geglühte $\mathrm{BaSO}_{4}$-Niederschlag nur schwefelsaures Baryum und Chlorbaryum enthält. Zieht man nun unsere oben angeführten Versuche mit Phenolphtalein in bezug auf alkalische Reaktion in Betracht, so scheint es sicher zu sein, dafs $\mathrm{zwischen} 650^{\circ} \mathrm{C}$. und etwa $900^{\circ} \mathrm{C}$. der $\mathrm{BaSO}_{4^{-}}$ Niederschlag neutral ist und das Übergewicht über $\mathrm{BaSO}_{4}$ von Chlorbaryum herrührt. Der $\mathrm{BaSO}_{4}$-Niederschlag fängt bei etwa $900^{\circ} \mathrm{C}$. an eine merkliche Menge Chlor zu verlieren und wird nun alkalisch.

\section{Eine Erklärung der obigen Resultate.}

Einige wichtige Beiträge unserer Kenntnisse der Stufenweisedissoziation von Verbindungen des Typus $\mathrm{MX}_{2}$ sind neulich veröffentlicht worden. C. L. v. ENDE ${ }^{1}$ stellt z. B. die Dissoziation des Chlorbleis folgendermassen dar:

$$
\begin{aligned}
& \mathrm{PbCl}_{2} \rightleftarrows \mathrm{PbCl}^{\cdot}+\mathrm{Cl}^{\prime} \\
& \mathrm{PbCl} \rightleftarrows \mathrm{Pb}^{*}+\mathrm{Cl}^{\prime} .
\end{aligned}
$$

Die Dissoziation tritt zwar stärker in der ersten Stufe als in der zweiten hervor. Bei der gesättigten wässerigen Lösung, die 38.8 Millimole Salz im Liter enthält, erfolgt die Dissoziation zu $50.1 \% \mathrm{~Pb}^{*}$ und $43.7 \% \mathrm{PbCl}$.

Bei seinen Untersuchungen über die Dissoziation der Merkurihaloide findet Monse, ${ }^{2}$ dals der unverbundene Teil des Merkurihaloids, falls das Haloidsalz in Merkurinitratlösung aufgelöst ist, das Kation $\mathrm{HgX}$ ' bis zu ziemlich starken Konzentrationen bildet, wo $\mathrm{X}=\mathrm{Cl}, \mathrm{Br}$, resp. J. Diese $\mathrm{HgX} \cdot$-Konzentration wird von der GröIsenordnung der gesamten Hg“-Ionen Konzentration geschätzt.

Betrachtet man nun die stufenweise Dissoziation von Chlorbaryum und Schwefelsäure, so hat man zunächst:

$$
\begin{aligned}
& \text { I. } \begin{array}{l}
\mathrm{BaCl}_{2} \rightleftarrows \mathrm{BaCl}^{\prime}+\mathrm{Cl}^{\prime}(\mathrm{a}) \text {, } \\
\mathrm{BaCl}^{\rightleftarrows} \rightleftarrows \mathrm{Ba}^{*}+\mathrm{Cl}^{\prime}(\mathrm{b}),
\end{array} \text { und } \\
& \text { II. } \mathrm{H}_{2} \mathrm{SO}_{4} \rightleftarrows \mathrm{H}+\mathrm{HSO}_{4}^{\prime} \text { (c). } \\
& \mathrm{HSO}_{4} \rightleftarrows \mathrm{H} \cdot+\mathrm{SO}_{4}^{\prime \prime} \text { (d). }
\end{aligned}
$$

1 Z. anorg. Chem. 21 (1899), 29.

${ }^{2}$ Zeitschr. phys. Chem. 41 (1902), 709. 
Es ist wohl wahrscheinlich, dals bedeutende Konzentrationen von $\mathrm{BaCl}^{\circ}$ und $\mathrm{HSO}_{4}^{\prime}$ in den Lösungen bestehen. Wenn aber Lösungen von Chlorbaryum und Schwefelsäure zusammengebracht sind, so würde sich etwas $\mathrm{BaCl} \mathrm{HSO}_{4}$ bilden können. $\mathrm{Ba} "$ - und $\mathrm{SO}_{4}{ }^{\prime \prime}$-Ionen werden natürlich bis zum Löslichkeitsprodukt von $\mathrm{BaSO}_{4}$ aus der Lösung verschwinden. Wenn aber $\mathrm{BaCl} \mathrm{HSO}_{4}$ zugegen ist, würde, falls es mit dem $\mathrm{BaSO}_{4}$ isomorph ist oder mit diesem eine feste Lösung bilden könnte, der Niederschlag aus den beiden Salzen bestehen. Die Entfernung von $\mathrm{Ba}^{*}-$ resp. $\mathrm{SO}_{4}{ }^{\prime \prime}$-Ionen würde wohl eine entsprechende Verminderung der $\mathrm{BaCl}$ - resp. $\mathrm{HSO}_{4}^{\prime}$-Ionen mit sich bringen. Aber es könnte doch etwas $\mathrm{BaCl}$. $\mathrm{HSO}_{4}$ vom Niederschlag mitgerissen werden. Beim Glühen würde dann der Niederschlag $\left(\mathrm{BaSO}_{4} ; \mathrm{BaCl} . \mathrm{HSO}_{4}\right)$ Salzsäure abgeben und der Rückstand aus neutralem $\mathrm{BaSO}_{4}$ bestehen. Man mufs aber auch die andere Möglichkeiten ins Auge fassen. Nämlich:

$$
\text { III. } 2 \mathrm{BaCl}^{\circ}+\mathrm{SO}_{4}^{\prime \prime} \rightleftarrows \underset{\mathrm{BaCl}}{\mathrm{BaCl}}>\mathrm{SO}_{4}
$$

und

$$
\text { IV. } 2 \mathrm{HSO}_{4}^{\prime}+\mathrm{Ba} * \underset{\mathrm{HSO}_{4}}{\mathrm{HSO}_{4}}>\mathrm{Ba} .
$$

Es ist übrigens nicht ausgeschlossen, dals Chlorbaryum im $\mathrm{BaSO}_{4}$-Niederschlag zugegen sein kann, da Chlorbaryum in demselben Kristallsystem mit schwefelsaurem Baryum vorkommt.

Das $\mathrm{Salz} \underset{\mathrm{BaCl}}{\mathrm{BaCl}}>\mathrm{SO}_{4}$ w wrde allein nicht im stande sein, Salzsäure abzugeben, und Schwefelsäure, welche sich aus $\mathrm{HSO}_{4}>\mathrm{Ba}$ bilden könnte, haben wir niemals konstatiert. Die beiden Salzpaare $\underset{\mathrm{BaCl}}{\mathrm{BaCl}}>\mathrm{SO}_{4}$ und $\mathrm{HSO}_{4}>\mathrm{Ba}$ würden aber zusammen Salzsäure bilden können, und in dem Falle würde neutrales $\mathrm{BaSO}_{4}$ übrig bleiben.

Wir haben nicht gesucht, Aufschlufs über die Anwesenheit von Wasser in der festen Phase zu geben. Das chlorfreie $\mathrm{BaSO}_{4}$ enthält wohl weniger Wasser als der von Chlorbaryum resp. Schwefelsäurelösungen ausgeschiedene Niederschlag. Es kann sein, dals einer oder sogar mehrere von den oben angeführten Salzen hydratisiert sind. Dals wir keine stöchiometrische Beziehung zwischen Wasser und dem gesamten Chlor finden konnten, spricht nicht gegen die letzte Auffassung.

Es gibt auch die Möglichkeit einer chemischen Reaktion zwischen Wasser und Chlorbaryum, welche Salzsäure frei machen würde. Der 
Rückstand wird in diesem Fall aber alkalisch reagieren. RICHARDs ${ }^{1}$ hat aber gezeigt, dafs der geglühte $\mathrm{BaSO}_{4}$-Niederschlag nur aus schwefelsaurem Baryum und Chlorbaryum besteht. Unsere oben angeführten Versuche in bezug auf diesen wichtigen Punkt lassen nun keinen Zweifel mehr bestehen, dafs der geglühte Niederschlag, welcher Salzsäure und Wasser verloren hat, neutral ist und sich aus schwefelsaurem Baryum und Chlorbaryum zusammensetzt, aber mit der Einschränkung, dafs die Temperatur etwa $900^{\circ} \mathrm{C}$. nicht übersteigt.

Unsere Versuche zeigen weiter, dafs der $\mathrm{BaSO}_{4}$-Niederschlag die Salzsäure als solche nicht mit sich zum Boden führt. Wenn $\mathrm{BaSO}_{4}$ aus einer Chlorbaryumlösung niedergeschlagen wird, welche immer ein bischen alkalisch mit Barytlösung erhalten wurde, so enthält der unter diesen Bedingungen ausgeschiedene Niederschlag immer fluchtiges sowie anch fixes Chlor.

Es schien uns, dafs weitere Beweise zunächst aus dem Verhalten einer Chlorbaryumlösung gegen den reinen chlorfreien $\mathrm{BaSO}_{4} \cdot \mathrm{Nieder}-$ schlag zu entnehmen wären. Werden nämlich die Substanzen, welche Salzsäure abgeben, vom chlorfreien festen schwefelsauren Baryum aus Chlorbaryumlösung aufgenommen?

Verdünnte Lösungen von Baryt und Schwefelsäure wurden bei $100^{\circ} \mathrm{C}$. sehr langsam zusammengebracht und zwei Tage lang rotiert, bis der Niederschlag grobkörnig geworden war. Dann wurde dieser chlorfreie $\mathrm{BaSO}_{4}$-Niederschlag in Chlorbaryumlösung $(v=1 \mathrm{~L}$. $)$, hineingebracht und 20 Tage bei $100^{\circ} \mathrm{C}$. weiter rotiert. Die ausgewaschene und bei $140^{\circ} \mathrm{C}$. getrocknete feste Phase enthielt jetzt kaum eine Spur Chlor.

Aus diesem Versuch ersieht man, dafs reines schwefelsaures Baryum, nachdem es grobkörnig geworden ist, die Substanzen welche das fixe sowie flüchtige Chlor liefern, nicht aufnimmt. Wir haben diesen Versuch wiederholt und immer denselben Ausgang bekommen. Wir halten den Versuch für einen Beweis, dafs die beobachteten Phänomene nicht von Oberflächenadsorption herrühren, und weiter, dafs unsere Methode, den Niederschlag auszuwaschen, befriedigend ist.

Bei noch einem anderen Versuch war chlorfreies schwefelsaures Baryum hergestellt und sogleich, wenn es immer noch feinkörnig war, in eine Chlorbaryumlösung hineingebracht. Das System wurde während 20 Tage bei $100^{\circ} \mathrm{C}$. rotiert. Der sorgfältig ausgewaschene

1 Richards, 1. c. 
und bei $140^{\circ} \mathrm{C}$. getrocknete Niederschlag enthielt jetzt 1.45 Mole Chlor zu 100 Mole schwefelsaures Baryum, also $0.22 \%$ Chlor. Eine andere Probe, welche 10 Tage bei $100^{\circ} \mathrm{C}$. rotiert, dann gut ausgewaschen und bei $140^{\circ} \mathrm{C}$. getrocknet wurde, ergab $0.135 \%$ Chlor. Der bei $650^{\circ}$ C. geglühte Rückstand enthielt $0.112 \%$ Chlor und es fand kein weiterer Verlust bis $850^{\circ} \mathrm{C}$. statt. Die bei den letzten zwei Versuchen erhaltenen Niederschläge haben daher einen viel kleinern Gehalt an flüchtigem Chlor im Verhältnis zum fixen, als der Niederschlag aus Chlorbaryumlösungen. Man wird wohl merken, dafs unter diesen Versuchsbedingungen eine verschwindend kleine Konzentration des Ions $\mathrm{HSO}_{4}{ }^{\prime}$ vorhanden ist, welche von der Löslichkeit des schwefelsauren Baryums herrührt.

C. L. v. ENDE ${ }^{1}$ hat bei seinen Versuchen gezeigt, dafs die gesättigte Lösung von Chlorblei $43 \% \mathrm{PbCl}$ enthält. Wir haben nun schwefelsaures $\mathrm{Blei}$ aus einer gesättigten $\mathrm{PbCl}_{2}$-Lösung niedergeschlagen und wir finden dabei, dafs der Niederschlag sowohl fixen wie flüchtigen Chlors in etwa demselben Verhältnis als der $\mathrm{BaSO}_{4}$ Niederschlag enthält.

Das Bedürfnis einer Entscheidung zwischen isomorphen Gemengen und festen Lösungen ist schon hervorgetreten. KüsteR ${ }^{2}$ glaubt, dals Diffusion allein in festen Lösungen möglich ist. Wenn aber die beigemengte Substanz am Bau eines isomorphen Kristalls teil nimmt, so ist seine Lage dadurch fixiert.

Es spricht sehr viel zu gunsten dieser Ansicht, dafs Diffusion das wahre Kennzeichen einer festen Lösung ist. Die geologischen Wissenschaften bieten zwar viele Beweise gegen die Idee, dals Diffusion in den wahren isomorphen Kristallen stattfindet. Wenn isomorphe Gemengen sich aus Lösungen ausscheiden, so zeigen die Konzentrationen in den festen and flüssigen Phasen weder keine befriedigende Übereinstimmung noch Tendenz, dem Massenwirkungsgesetz Folge zu leisten, obwohl die elektrolytische Dissoziation mit

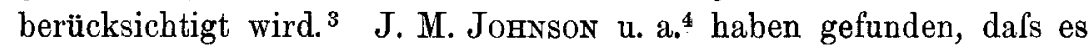
sehr schwierig ist, Mischkristalle von derselben Zusammensetzung zu bekommen.

${ }^{1}$ C. L. v. Ende, Z. anorg. Chem. 21 (1899), 29.

2 Zeitschr. phys. Chem. 17 (1896), 308.

${ }^{3}$ Fock, Zeitschr. f. Kristall. 28 (1897), 337.

4 J. M. Johnson, Journ. Chem. Soc. 35 (1879), 205. - Moнr, Ztitschr. phys. Chem. 27 (1898), 204. - Stortendecker, Zeitschr. phys. Chem. 43 (1903), 629. - Foote, Am. Chem. Journ. 26 (1902), 402. 
Im Falle aber, dafs Diffusion in der festen Phase stattfindet, stimmen diese Resultate untereinander überein. Sie leisten wohI dem Nernstschen Verteilungsgesetz Folge, wie Küster ${ }^{1}$ bei seinen interessanten Untersuchungen über das gleichzeitige Fällen von $\mathrm{AgCl}$ und $\mathrm{AgBr}$ gezeigt hat. Seien diese beiden Haloidsalze aus einer Lösung zusammengefällt oder sei jedes einzeln gefällt und erst dann die Vermischung bewerkstelligt, es war immer dasselbe Endresultat nach wenigen Stunden erreicht. Seine Erfahrungen bekunden eine Übereinstimmung mit dem Verteilungsgesetz, also sicher eine feste Lösung. Unser Fall ist gerade das Gegenteil von diesem. Der während 20 Tage bei $100^{\circ} \mathrm{C}$. rotierte, schon grobkörnig gewordene Niederschlag nahm keine melsbare Menge Chlor aus Chlorbaryumlösung auf. Wenn aber reines, feinkörniges schwefelsaures Baryum in Chlorbaryumlösung eingebracht wird und erst dann die Partikelchen den grobkörnigen Zustand annehmen, werden merkliche Mengen von Chlor aufgenommen.

Man bätte wohl glauben können, dals das Zeitelement, welches wir oben angeführt haben, auf eine mögliche Diffusion von Chlorbaryum aus der festen Phase deutet. Wir haben nun immer beobachtet, dafs der feinkörnige $\mathrm{BaSO}_{4}$-Niederschlag immer mehr Chlor enthält, als der schon grobkörig gewordene. Wir glauben aber, dafs diese Änderung im Chlorgehalt eine andere Ursache hat. Da die feinkörnigen Kristalle bedeutend löslicher sind als die grobkörnigen, ${ }^{2}$ so werden sie aufgelöst, und die so entstandene übersättigte Lösung wird dann schwefelsaures Baryum auf die grölseren Kristalle ausscheiden. Im allgemeinen wird die Zusammensetzung irgend eines Mischkristalles von den relativen Löslichkeiten seiner Bestandteile abhängen. Je löslicher das schwefelsaure Baryum, desto weniger schwefelsaures Baryum und desto mehr vom anderen Bestandteil finden sich im Mischkristalle. Wir würden demgemäls eine gröfsere Menge von den beigemengten Substanzen in den feinkörnigeren Niederschlägen erwarten. Da aber die feinkörnigen Kristallchen sich auflösen und nachher auf den grölseren Kristallen sich abscheiden, so sehen wir ein, wie der Chlorgehalt sich allmählich ändert, gemäls unsern oben mitgeteilten Beobachtungen. Deshalb stimmen unsere Beobachtungen über das Zeitelement mit der Idee, dafs eine Diffusion im Mischkristalle nicht stattfindet, überein.

1 Z. anorg. Chem. 19 (1899), 81.

${ }^{2}$ Houetr, Zeitschr. phys. Chem. 37 (1901), 400. 
Wenn unsere Erklärung der Tatsachen die richtige ist, so rühren die Stoffe, welche mit dem schwefelsauren Baryum niedergeschlagen sind, von der Anwesenheit der zwei Ionen $\mathrm{BaCl}^{-}$und $\mathrm{HSO}_{4}{ }^{\prime}$ her. Wir würden also reines schwefelsaures Baryum bekommen, wenn diese zwei Ionen abwesend wären. Diese Bedingungen sind getroffen, falls das Baryumsalz einer zweibasischen Säure mit dem schwefelsauren Salz eines zweiwertigen Metalls, etwa $\mathrm{MgSO}_{4}$, zusammen in eine Lösung gebracht sind. Wir hatten zur Hand etwas aus sehr reinen Materialien hergestelltes $\mathrm{BaPt}(\mathrm{CN})_{4}$. $4 \mathrm{H}_{2} \mathrm{O}$, welches mehrmals umkristallisiert war.

Ausgewogene Mengen dieses Salzes wurden mit einem grolsen Überschufs von $\mathrm{MgSO}_{4}$-Lösung niedergeschlagen. Folgende Tabelle ergibt die Versuchsergebnisse.

$\begin{array}{ccc}\mathrm{BaPt}(\mathrm{CN})_{4} \cdot 4 \mathrm{H}_{2} \mathrm{O} & \begin{array}{c}\mathrm{BaSO}_{4} \\ \text { gefunden: }\end{array} & \begin{array}{c}\mathrm{BaSO}_{4} \\ \text { berechnet: }\end{array} \\ 0.9876 \mathrm{~g} & 0.4530 \mathrm{~g} & 0.4534 \mathrm{~g} \\ 1.2248 \mathrm{~g} & 0.5619 \mathrm{~g} & 0.5625 \mathrm{~g} \\ 1.1066 \mathrm{~g} & 0.5077 \mathrm{~g} & 0.5083 \mathrm{~g} \\ 2.0035 \mathrm{~g} & 0.9199 \mathrm{~g} & 0.9200 \mathrm{~g} \\ 2.0921 \mathrm{~g} & 0.9609 \mathrm{~g} & 0.9607 \mathrm{~g}\end{array}$

Bei den letzten zwei Versuchen wurden die $\mathrm{BaPt}\left(\mathrm{CN}_{4}\right)_{4} .4 \mathrm{H}_{2} \mathrm{O}$. Lösungen in überschüssiger $\mathrm{MgSO}_{4}$-Lösung eingegossen und das $\mathrm{BaSO}_{4}$ im Waschwasser wieder gewonnen und mitberechnet. Bei den ersten drei Versuchen waren aber die $\mathrm{MgSO}_{4}$-Lösungen der Ba-Salzlösung hinzugesetzt und nichts im Waschwasser berücksichtigt. Die Übereinstimmung der gefundenen mit den theoretischen Werten lärst also nichts zu wünschen übrig. Der geglühte Niederschlag sah schneeweils aus und war pulverförmig. Er war nicht in einer festen Masse zusammengnschrumpft, wie es bei unreinen $\mathrm{BaSO}_{4}$ Niederschlägen gewöhnlich der Fall ist.

Sämtliche zur Zeit bekannten, den $\mathrm{BaSO}_{4}$-Niederschlag betreffenden Tatsachen finden vielleicht auch andere Erklärungen. Unserer Meinung nach ist die oben gegebene die geeignetste. Unsere zuerst gehegte Ansicht, diese Phänomena auf eine Oberfächenadsorption und Okklusion zurückzufübren, haben wir im Laufe der Arbeit aufgegeben, weil manche Beobachtungen dagegen sprechen, so z. B. die Tatsache, dafs die Diffusionskristalle des schwefelsauren Baryums sich äbnlich einem $\mathrm{BaSO}_{4}$-Niederschlag verhalten, welcher momentan gebildet wird und relativ eine viel grölsere Oberfläche besitzt als jene. Einen besonderen Hinweis gegen Adsorption liefert 
die Beobachtung, dafs der reine grobkörnige $\mathrm{BaSO}_{4}$-Niederschlag aus einer Chlorbaryumlösung nichts aufnimmt. Man beobachtet aufserdem, dals diese Fähigkeit des schwefelsauren Baryums andere Substanzen mit sich zum Boden zu führen selektiv ${ }^{1}$ ist. Unser Versuch mit $\mathrm{MgSO}_{4}$ und $\mathrm{BaPt}(\mathrm{CN})_{4} \cdot 4 \mathrm{H}_{2} \mathrm{O}$ ergibt einen Niederschlag, der nichts mit sich zum Boden führt.

Isomorphismus, wo der eine Bestandteil nur spurenweise zugegen ist, welches auf dem Umstand beruht, dafs dieser Bestandteil leicht löslich, der andere schwerlöslich ist, tritt wahrscheinlich viel öfter ein, als zurzeit geglaubt wird. Die vielen Unregelmälsigkeiten, die bei der Analyse auftreten, dürften wohl in diesem Umstand ihre Erklärung finden. Wir hoffen auf diese interessante Frage zurückzukommen.

Wir kommen hiernach zu dem Schlufs, dafs $\mathrm{Ba}$ - oder $\mathrm{SO}_{4}$ "Ionen sich in der Abwesenheit $\mathrm{BaX}$ - resp. $\mathrm{MSO}_{4}^{\prime}$-Ionen genau bestimmen lassen müssen, haben aber noch kein geeignetes Baryumsalz einer zweibasischen Säure gefunden. $\mathrm{MgSO}_{4}$ ist allerdings befriedigend.

Der Vorschlag Richa RDs, dafs man das Chlor im geglühten $\mathrm{BaSO}_{4}$-Niederschlag bestimmt und die entsprechende Menge von Chlorbaryum abzieht, um richtige Resultate zu bekommen bei der Bestimmung von $\mathrm{SO}_{4}$ " mit einem Überschuls von Chlorbaryum, findet Bestätigung durch unsere Arbeit. Unsere Methode bietet zwar ein einfaches und sehr genaues Mittel, das Chlor mit einem leicht zu konstruierenden Apparat zu bestimmen. Wir schliefsen aber, dafs der $\mathrm{BaSO}_{4}$-Niederschlag nicht über Rotglühhitze (etwa $700^{\circ} \mathrm{C}$.), geglüht werden darf, und das Filter sollte nach der Methode von BUNSEN $^{2}$ unterhalb Rotglühhitze zu Asche verbrannt werden. Falls $\mathrm{SO}_{4}$ " mit einem Überschuls von Chlorbaryum zu bestimmen ist, mufs man das im Niederschlag enthaltene Chlorbaryum vom Gewicht des geglühten Niederschlages abziehen. Wenn aber $\mathrm{Ba}$ " mit einem Überschufs von Schwefelsäure bestimmt wird, ist das vorhandene Chlorbarium als schwefelsaures Baryum zu rechnen und zu dem im Niederschlag gefundenen schwefelsauren Baryum zuzusetzen, da das Gewicht des Niederschlages in diesem Falle zu klein ausfällt.

1 Vergl. auch Fresenide, Zeitschr. analyt. Chem. 9 (1870), 52.

${ }^{2}$ Zeitschr. analyt. Chem. 8 (1869), 187.

Ann. Arbor, University of Michigan, Chemical Laboratory.

Bei der Redaktion eingegangen am 29. April 1904. 